DOI: $10.17516 / 1998-2836-0203$

УДК $543.545,54.04$

\title{
Determining Stability Constants \\ of Complexes Between Ester Betulin Derivatives \\ and $\beta$-Cyclodextrin by Affinity Capillary Electrophoresis
}

\author{
Viktoria V. Sursyakova*a, \\ Vladimir A. Levdansky and Anatoly I. Rubaylo ${ }^{\mathrm{a}, \mathrm{b}}$ \\ anstitute of Chemistry and Chemical Technology SB RAS \\ FRC "Krasnoyarsk Science Center SB RAS" \\ Krasnoyarsk, Russian Federation \\ ${ }^{b}$ Siberian Federal University \\ Krasnoyarsk, Russian Federation
}

Received 02.10.2020, received in revised form 05.11.2020, accepted 03.12.2020

Abstract. Inclusion complexes of ester betulin derivatives (pentacyclic lupane triterpenoids) with $\beta$-cyclodextrin $(\beta-C D)$ were studied by affinity capillary electrophoresis. Stability constants were calculated from dependencies of viscosity-corrected effective electrophoretic mobilities on $\beta$-CD concentration (0-10 $\mathrm{mM})$ in background electrolytes using nonlinear regression fitting. Logarithms of stability constants at $25{ }^{\circ} \mathrm{C}$ for 1:1 and 1:2 $\beta$-CD complexes of betulin 3,28-diphthalate, betulin 3,28-disuccinate and betulin 3,28-disulfate (95\% confidence interval) are 4.25 (4.16-4.32) and 7.27 (6.73-7.50), 4.38 (4.26-4.48) and 7.58 (6.90-7.84), 4.04 (4.00-4.08) and 5.91 (4.60-6.20), respectively.

Keywords: drug delivery, host-guest complexes, capillary electrophoresis, binding constants, inclusion complexes.

Citation: Sursyakova V.V., Levdansky V.A., Rubaylo A.I. Determining stability constants of complexes between ester betulin derivatives and $\beta$-cyclodextrin by affinity capillary electrophoresis, J. Sib. Fed. Univ. Chem., 2020, 13(4), 534-541. DOI: $10.17516 / 1998-2836-0203$

(C) Siberian Federal University. All rights reserved

This work is licensed under a Creative Commons Attribution-NonCommercial 4.0 International License (CC BY-NC 4.0).

* Corresponding author E-mail address: viktoria_vs@list.ru 


\title{
Определение констант устойчивости \\ комплексов эфирных производных бетулина \\ с $\beta$-циклодекстрином методом \\ аффинного капиллярного электрофореза
}

\author{
В.В. Сурсякова ${ }^{a}$, В.А. Левданский ${ }^{\mathrm{a}}$, А.И. Рубайло \\ ${ }^{a}$ Институт химии и химической технологии СО РАН \\ ФИЦ «Красноярский научный иентр СО РАН» \\ Российская Федерация, Красноярск \\ ${ }^{\sigma}$ Сибирский федеральный университет \\ Российская Федераиия, Красноярск
}

Аннотация. Исследованы комплексы включения, образованные эфирными производными бетулина (пентациклическими тритерпеноидами лупанового ряда) и $\beta$-циклодекстрином ( $\beta$-ЦД), с применением аффинного капиллярного электрофореза. Методом нелинейной регрессии из полученных зависимостей электрофоретических подвижностей от концентрации $\beta$-ЦД (0-10 мМ) в фоновых электролитах найдены константы устойчивости. Значения логарифмов констант устойчивости для 1:1 и 1:2 $\beta$-ЦД комплексов при $25{ }^{\circ} \mathrm{C}$ для 3,28-дифталата, 3,28-дисукцината и 3,28-дисульфата бетулина (95 \% доверительный интервал) составили 4.25 (4.16-4.32) и 7.27 (6.73-7.50), 4.38 (4.26-4.48) и 7.58 (6.90-7.84), 4.04 (4.00-4.08) и 5.91 (4.60-6.20) соответственно.

Ключевые слова: доставка лекарств, комплексы хозяин-гость, капиллярный электрофорез, константы связывания, комплексы включения.

Цитирование: Сурсякова, В.В. Определение констант устойчивости комплексов эфирных производных бетулина с $\beta$-циклодекстрином методом аффинного капиллярного электрофореза / В.В. Сурсякова, В.А. Левданский, А.И. Рубайло // Журн. Сиб. федер. ун-та. Химия, 2020. 13(4). С. 534-541. DOI: 10.17516/1998-2836-0203

\section{Введение}

Бетулин и его производные, пентациклические тритерпеноиды лупанового ряда, обладают рядом полезных свойств (противовирусная, противораковая и другая активность) и широко распространены в природе [1]. Однако их молекулы достаточно гидрофобны, вследствие чего бетулин и некоторые его производные плохо растворимы в воде. Для повышения растворимости и биодоступности часто используют комплексы включения (комплексы хозяин-гость, супрамолекулярные комплексы) таких гидрофобных веществ с циклодекстринами. Циклодекстрины (ЦД) [2] - природные циклические олигосахариды, построенные из остатков $\alpha-1,4-$ связанных D-глюкопираноз. Наиболее распространенными являются молекулы ЦД с 6, 7 и 8 остатками глюкозы, которые называются $\alpha-, \beta$ - и $\gamma$-ЦД соответственно. Они характеризуются уникальной структурой: молекула имеет форму усеченного конуса, внутренняя полость которого гидрофобна, благодаря наличию этой полости ЦД способны образовывать с различными 
соединениями комплексы включения. В этих комплексах ЦД выступает как молекула хозяина, а различные соединения являются гостевыми молекулами.

Одним из современных методов с небольшим расходом реагентов для изучения ЦД комплексов считается метод аффинного капиллярного электрофореза (АКЭ). Наиболее распространенный его вариант основан на измерении электрофоретической подвижности гостевых молекул (аналита) в фоновых электролитах с различным содержанием ЦД. На основе следующего ниже уравнения методом нелинейной регрессии определяют константы устойчивости:

$$
v \cdot \mu_{э_{э \phi \phi}}=\frac{\mu_{0}+\mu_{11} \beta_{11}[Ц Ц]_{i}+\mu_{12} \beta_{12}[Ц Ц]_{i}^{2}}{1+\beta_{11}[Ц Ц]_{i}+\beta_{12}[Ц Д]_{i}^{2}},
$$

где $v$ - коэффициент для коррекции изменения вязкости фонового электролита; $\mu_{\text {эфф }}$ - эффективная электрофоретическая подвижность; $\mu_{0}, \mu_{11}$ и $\mu_{12}$ - ионные подвижности исследуемого соединения, 1:1 и 1:2 комплекса соответственно; $\beta_{11}$ и $\beta_{12}$ - константы устойчивости 1:1 и 1:2 комплекса; [ЦД] - концентрация ЦД в фоновом электролите.

Ранее были изучены комплексы ряда производных бетулина (бетулиновая и бетулоновая кислоты, 3,28-дифталат и 3,28-дисукцинат бетулина, сульфатированные производные бетулина) с $\beta$-ЦД, гидроксипропил- $\beta$ - и $\gamma$-ЦД (ГП- $\beta$-ЦД и ГП- $\gamma$-ЦД) [3-10]. Для исследования этих комплексов с практически нерастворимыми соединениями был предложен новый метод, основанный на комбинации метода растворимости и капиллярного зонного электрофореза [5]. Также в процессе исследования ГП- $\gamma$-ЦД комплексов были рассмотрены особенности применения АКЭ для определения достаточно больших значений констант устойчивости циклодекстриновых комплексов $\mathrm{c} \log \beta_{11} \sim 7$; обычно логарифмы констант устойчивости для ЦД комплексов находятся в диапазоне 1-4 [8, 9]. Показано, что для ГП- $\beta$-ЦД характерно наличие 1:1 и 1:2 комплексов с производными бетулина [6] в отличие от ГП- $\gamma$-ЦД, для которого характерны только 1:1 комплексы $[8,9]$.

Цель данной работы - исследование $\beta$-ЦД комплексов эфирных производных бетулина методом АКЭ. Комплексы 3,28-дифталата (ДФБ) и 3,28-дисукцината бетулина (ДСкБ) с $\beta$-ЦД впервые изучены в рамках данной работы, в то время как $\beta$-ЦД комплексы 3,28-дисульфата бетулина (ДСБ) уже ранее исследовались, но в ограниченном концентрационном диапазоне $\beta$-ЦД, и была определена константа устойчивости только для 1:1 комплекса [3].

\section{Материалы и методы}

В работе использовали реактивы марки ч.д.а. или х.ч. Растворы готовили из деионизованной воды (система очистки воды Direct Q3, Millipore, France) и фильтровали через 0.45 мкм фильтры. $\beta$-ЦД ( > 98 \%) был приобретен в Acros Organics (Belgium). Раствор 10 мМ тетрабората натрия с рН 9.18 с добавками 0-10 мМ $\beta$-ЦД был применен как фоновый электролит, $\beta$-ЦД растворяли непосредственно в растворе тетрабората натрия. В качестве маркера электроосмотического потока (ЭОП) использовали 0.001 \% диметилсульфоксид (ДМСО). Производные бетулинов синтезированы как описано в работах [11-13]. Растворы производных бетулина с концентрацией 1 г/л готовили путем растворения точных навесок в 10 мМ тетраборате натрия. Образцы аналитов для ввода в капилляр готовили путем разбавления концентрированных растворов соответствующим фоновым электролитом.

$$
-536-
$$


В работе использовали прибор Красноярского регионального центра коллективного пользования ФИЦ КНЦ СО РАН - систему капиллярного электрофореза с диодно-матричным детектором Agilent 7100 (Agilent Technologies, Waldbronn, Germany). Применяли немодифицированный кварцевый капилляр с внутренним диаметром 50 мкм, общей и эффективной длиной $80.5 / 72$ см. Капилляр термостатировали при $25 \pm 0.04{ }^{\circ} \mathrm{C}$. Детектирование проводили в УФобласти при 200 нм. Разделение осуществляли при +30 кВ. Образцы вводили в капилляр гидродинамически при давлении 50 мбар в течение 5 с. Все эксперименты проводили в 3-5 параллелях. В начале каждого дня капилляр промывали $0.1 \mathrm{M} \mathrm{NaOH}$ в течение 10 мин, дважды водой по 5 мин, затем фоновым электролитом в течение 10 мин. Между съемками капилляр промывали фоновым электролитом в течение 3 мин.

Эффективная электрофоретическая подвижность из экспериментальных данных рассчитывалась следующим образом:

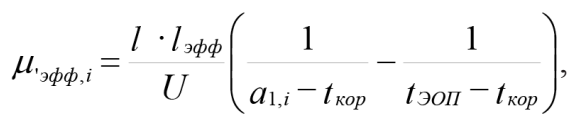

$$
\begin{aligned}
& t_{\text {кор }}=\left(t_{\text {окон }}+t_{\text {нач }}\right) / 2 \text {, }
\end{aligned}
$$

где $l$ и $l_{\text {эфф }}$ - общая и эффективная длина капилляра; $U$ - напряжение; $a_{l, i}$ - центр пика функции Хаархова-Ван-дер Линде (ХВЛ, Нaarhoff-Van der Linde function) для $i$ пика [14]; $t_{\text {кор }}$ - коррекционная поправка времени, учитывающая то, что напряжение прикладывается

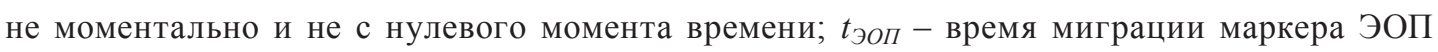
(нейтрального соединения - ДМСО); $t_{\text {нач }}$ время, с которого начинает прикладываться напряжение $\left(0.01\right.$ мин); $t_{\text {окон }}$ - время, при котором напряжение достигает заданного значения (0.18 мин).

Коэффициент для коррекции изменения вязкости фонового электролита рассчитывали следующим образом $[9,10]$ :

$$
v=t^{\prime} / t^{0}
$$

где $t^{\prime}$ и $t^{0}$ - времена ДМСО пика, полученные при напряжении 0 кВ и давлении 100 мбар в фоновых электролитах с добавкой $\beta$-ЦД и без нее соответственно.

Константы устойчивости комплексов и ионные подвижности рассчитывали путем нелинейной регрессии с использованием MS Excel и OriginPro 8.1 (OriginLab Corporation, Northampton, USA) [8]. Параметр $a_{1}$ ХВЛ функции для электрофоретических пиков находили с применением программ MS Excel и CEval (Prague, Czech Republic [15]).

\section{Результаты и их обсуждение}

Были зарегистрированы электрофореграммы производных бетулина с использованием фоновых электролитов с концентрацией $\beta$-ЦД от 0 до 10 мМ. Брали минимально возможную концентрацию аналитов - такую, чтобы отношение сигнал/шум пиков равнялось 10. Для исследованных соединений это 0.002, 0.02 и 0.07 мМ для ДФБ, ДСБ и ДСкБ соответственно. На рис. 1 п приведен пример электрофореграмм.

По уравнениям (2)-(4) были рассчитаны эффективные электрофоретические подвижности. При наличии $\beta$-ЦД в фоновом электролите пики были треугольными и для них при расчете

$$
-537-
$$


a)

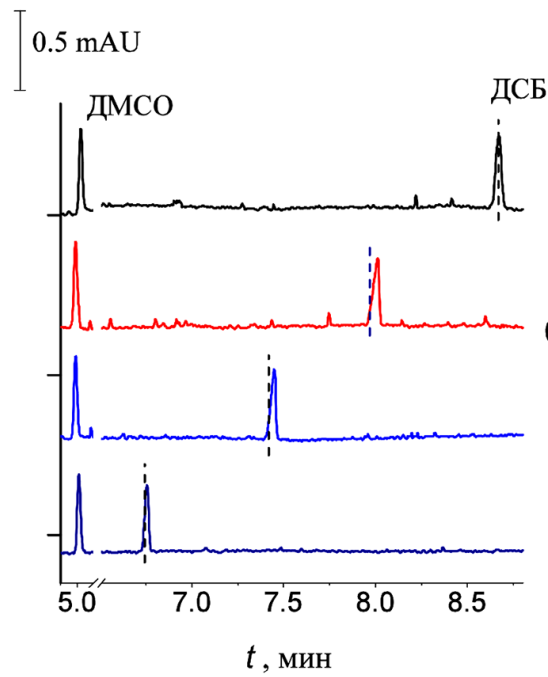

B)

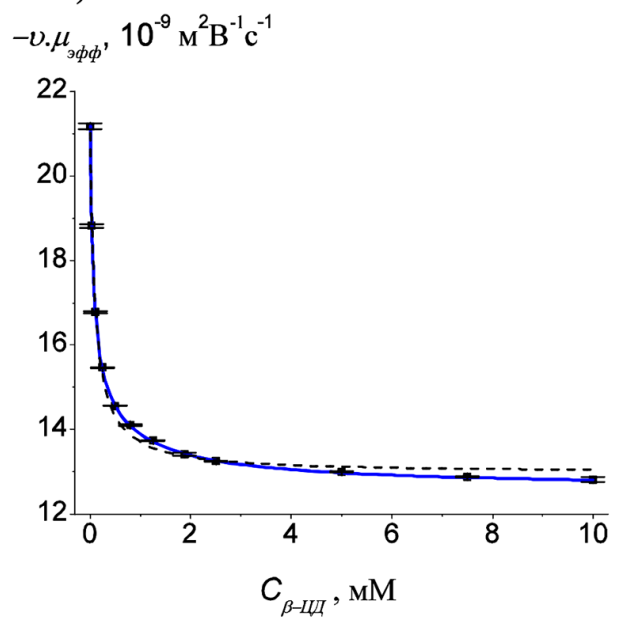

б)

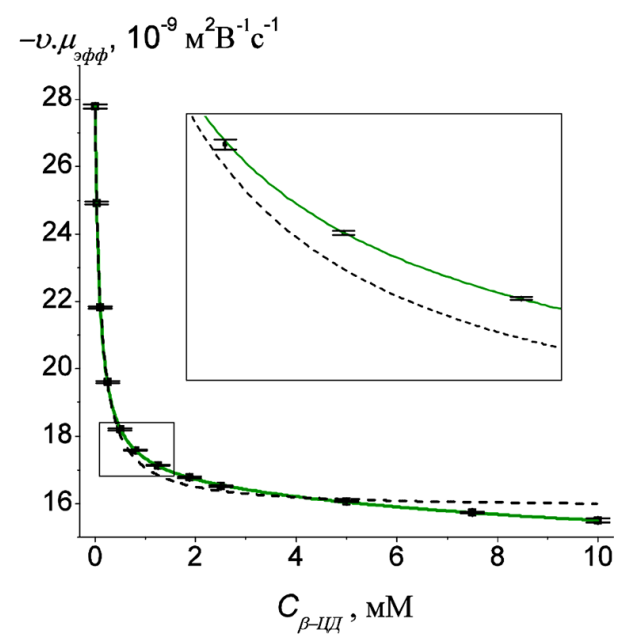

г)

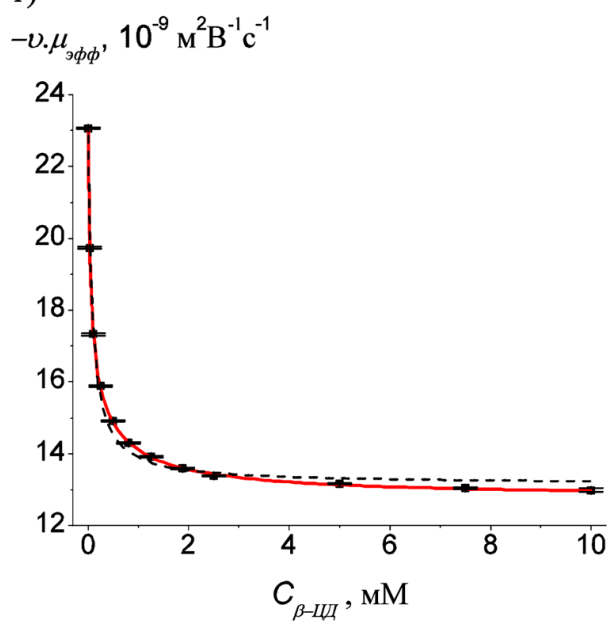

Рис. 1. Примеры электрофореграмм ДСБ, полученные при разном содержании $\beta$-ЦД в фоновом электролите $(a)$, и зависимости эффективной электрофоретической подвижности, скорректированной на изменение вязкости, от концентрации $\beta$-ЦД в фоновом электролите $(\sigma, 2)$. Аналиты (концентрация): $\sigma$ - ДСБ (0.02 мМ); в - ДФБ (0.002 мМ); г - ДСкБ (0.07 мМ). В блоке а) вертикальной пунктирной линией показано время, соответствующее $a_{1}$ параметру ХВЛ функции. В блоках б)-г) точки соответствуют экспериментальным значениям, сплошные линии - теоретическим зависимостям, учитывающим 1:1 и 1:2 комплексообразование, пунктирные линии - теоретическим зависимостям, учитывающим только 1:1 комплексообразование. Планки погрешностей представляют собой стандартное отклонение соответствующих величин. В прямоугольнике показаны точки, планки погрешностей и теоретические зависимости в увеличенном масштабе

Fig. 1. Examples of electropherograms of betulin 3,28-disulfate obtained using background electrolytes with different $\beta$-CD content (a) and dependencies of viscosity-corrected effective electrophoretic mobilities on $\beta$-CD concentration in background electrolytes (б-г). Analyte (concentration): б) betulin 3,28-disulfate $(0.02 \mathrm{mM})$, в) betulin 3,28-diphthalate $(0.002 \mathrm{mM}), \Gamma)$ betulin 3,28-disuccinate $(0.07 \mathrm{mM})$. In section a), the vertical dashed lines show the time corresponding to the $a_{1}$ parameter of the Haarhoff-Van der Linde function. In sections б)-г), the points are experimental values, the solid lines correspond to the theoretical curves taking into account 1:1 and 1:2 complexation, and the dashed lines correspond to the theoretical curves taking into account only 1:1 complexation. The error bars represent standard deviations of corresponding values. In a rectangle on б), the points, error bars and theoretical curves are shown on a larger scale 
подвижности использовали $a_{1}$ параметр ХВЛ функции, как описано в работах $[8,9]$. На рис. $1 a$ время, отвечающее $a_{l}$ параметру, обозначено пунктирной линией. Видно, что для треугольных пиков отличие может быть довольно значительным. Также в работе [9] показано, что значения констант устойчивости могут быть занижены на 50-70 \%, если использовать вместо $a_{l}$ параметра время, отвечающее максимуму пика.

На рис. 1б-г видно, что полученные экспериментальные точки не описываются теоретическими кривыми, учитывающими только 1:1 взаимодействие (пунктирные линии). На основе рассчитанных подвижностей методом нелинейной регрессии рассчитаны значения констант устойчивости для 1:1 и 1:2 комплексов. Как можно видеть на рис. 16-г, теоретические кривые, учитывающие образование 1:1 и 1:2 комплексов, хорошо согласуются с экспериментальными точками. Рассчитанные значения констант устойчивости и ионных подвижностей приведены в табл. 1. Как видим по данным табл. 1, ДФБ и ДСкБ имеют близкие с учетом погрешности значения констант устойчивости, в то время как константы устойчивости для ДСБ несколько ниже. Как и в случае комплексов этих соединений с ГП- $\gamma$-ЦД [9], это можно объяснить небольшим сродством фталатных и сукцинатных групп к внутренней гидрофобной полости ЦД. Кроме того, полученное значение константы устойчивости для ДСБ отличается от значения, полученного ранее, но в предыдущем исследовании использовался достаточно узкий диапазон концентраций $\beta$-ЦД (до 2 мМ по сравнению с 10 мМ, см. рис. 1б) и линеаризационный способ «двойных обратных величин», который, как было показано в [10], не позволяет выявить наличие 1:2 комплексов.

Таблица 1. Значения констант устойчивости для 1:1 и 1:2 комплексов исследованных соединений с $\beta$-ЦД и ионные подвижности соответствующих анионов при $25^{\circ} \mathrm{C}$. В скобках или после « » указан $95 \%$ доверительный интервал

Table 1. Stability constants for $1: 1$ and 1:2 complexes of the compounds under study with $\beta$-CD and ionic mobilities of corresponding species. In brackets or after « \pm », the $95 \%$ confidence interval is shown

\begin{tabular}{|c|c|c|c|c|c|c|}
\hline \multirow{2}{*}{ Аналит ${ }^{\mathrm{a}}$} & \multirow{2}{*}{$\log \beta_{11}$} & \multirow{2}{*}{$\log \beta_{12}$} & \multicolumn{3}{|c|}{ Ионные подвижности $\left(10^{-9} \mathrm{~m}^{2} \mathrm{~B}^{-1} \mathrm{c}^{-1}\right)$} & \multirow{2}{*}{ Ссылка } \\
\hline & & & $-\mu_{0}$ & $-\mu_{11}$ & $-\mu_{12}$ & \\
\hline \multirow[t]{2}{*}{ ДСБ } & $\begin{array}{c}4.04 \\
(4.00-4.08)\end{array}$ & $\begin{array}{c}5.91 \\
(4.60-6.20) \\
\end{array}$ & $27.8 \pm 0.1$ & $16.7 \pm 0.2$ & $13.7 \pm 1.9$ & $\begin{array}{l}\text { Данная } \\
\text { работа }\end{array}$ \\
\hline & $3.87 \pm 0.01^{\sigma}$ & $-{ }^{\text {в }}$ & $-{ }^{-}$ & $-{ }^{-r}$ & $-{ }^{-\mathrm{r}}$ & [3] \\
\hline ДФБ & $\begin{array}{c}4.25 \\
(4.16-4.32) \\
\end{array}$ & $\begin{array}{c}7.27 \\
(6.73-7.50) \\
\end{array}$ & $21.2 \pm 0.1$ & $14.8 \pm 0.7$ & $12.6 \pm 0.1$ & \multirow{2}{*}{$\begin{array}{c}\text { Данная } \\
\text { работа }\end{array}$} \\
\hline ДСкБ & $\begin{array}{c}4.38 \\
(4.26-4.48)\end{array}$ & $\begin{array}{c}7.58 \\
(6.90-7.84)\end{array}$ & $23.1 \pm 0.1$ & $15.8 \pm 1.0$ & $12.8 \pm 0.1$ & \\
\hline АСБ & $4.00 \pm 0.02^{6}$ & $-{ }^{\text {в }}$ & $-{ }^{-\mathrm{r}}$ & $-{ }^{-r}$ & $-{ }^{-\mathrm{r}}$ & [3] \\
\hline
\end{tabular}

аДСБ - 3,28-дисульфат бетулина, ДФБ - 3,28-дифталат бетулина, ДСкБ - 3,28-дисукцинат бетулина, АСБ - 3-ацетат-28-сульфат бетулина.

${ }^{\sigma}$ Для расчета доверительного интервала использован другой, отличный от используемого в данной статье, подход.

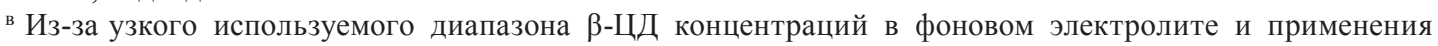
линеаризационного способа «двойных обратных величин» константы устойчивости для 1:2 комплексов не были определены.

г В тексте статьи [3] ионные подвижности не упомянуты. 


\section{Заключение}

Таким образом, методом аффинного капиллярного электрофореза исследовано комплексообразование 3,28-дифталата, 3,28-дисукцината и 3,28-дисульфата бетулина с $\beta$-ЦД. Установлено, что полученные зависимости подвижностей описываются с учетом образования комплексов с 1:1 и 1:2 стехиометрией. Рассчитаны значения констант устойчивости этих комплексов.

\section{Благодарности / Acknowledgements}

Работа выполнена в рамках государственного задания Института химии и химической технологии СО РАН (проект АААА-А17-117021310221-7) с использованием оборудования Красноярского регионального центра коллективного пользования ФИЦ КНЦ СО РАН.

This work was conducted within the framework of the budget project AAAA-A17-117021310221-7 for Institute of Chemistry and Chemical Technology SB RAS using the equipment of Krasnoyarsk Regional Research Equipment Centre of SB RAS.

\section{Список литературы / References}

1. Tolstikova T.G., Sorokina I.V., Tolstikov G.A., Tolstikov A.G., Flekhter O.B. Biological activity and pharmacological prospects of lupane terpenoids: I. Natural lupane derivatives. Russian Journal of Bioorganic Chemistry 2006. Vol. 32 (1), P. 37-49.

2. Crini G. Review: A history of cyclodextrins. Chemical Review 2014. Vol. 114, P. 1094010975.

3. Popova O.V., Sursyakova V.V., Burmakina G.V., Levdansky V.A., Rubaylo A.I. Determination of stability constants of inclusion complexes of betulin derivatives with $\beta$-cyclodextrin by capillary electrophoresis. Doklady Chemistry 2015. Vol. 461(1), P. 67-69.

4. Попова О.В., Сурсякова В.В., Бурмакина Г.В., Максимов Н.Г., Левданский В.А., Рубайло А.И. Исследование растворимости бетулоновой кислоты в присутствии гидроксипропил$\gamma$-циклодекстрина методом капиллярного электрофореза. Журнал Сибирского федерального университета. Химия 2016. Т. 9(2), C. 171-176. [Popova O.V., Sursyakova V.V., Burmakina G.V., Maksimov N.G., Levdansky V.A., Rubaylo A.I. Solubility study of betulonic acid in the presence of hydroxypropyl- $\gamma$-cyclodextrin by capillary electrophoresis. Journal of Siberian Federal University. Chemistry 2016. Vol. 9(2), P. 171-176. (In Russ.)].

5. Sursyakova V.V., Maksimov N.G., Levdansky V.A., Rubaylo A.I. Combination of phasesolubility method and capillary zone electrophoresis to determine binding constants of cyclodextrins with practically water-insoluble compounds. Journal of Pharmaceutical and Biomedical Analysis 2018. Vol. 160, P. 12-18.

6. Sursyakova V.V., Levdansky V.A., Rubaylo A.I. Thermodynamic parameters for the complexation of water-soluble betulin derivatives with (2-hydroxypropyl)- $\beta$-cyclodextrin determined by affinity capillary electrophoresis. Journal of Molecular Liquids 2019. Vol. 283, P. 325-331.

7. Sursyakova V.V., Levdansky V.A., Rubaylo A.I. Thermodynamic parameters for the complexation of water-insoluble betulin derivatives with (2-hydroxypropyl)- $\gamma$-cyclodextrin determined by phasesolubility technique combined with capillary zone electrophoresis. Electrophoresis 2019. Vol. 40(1213), P. 1656-1661. 
8. Sursyakova V.V., Levdansky V.A., Rubaylo A.I. Strong complexation of water-soluble betulin derivatives with (2-hydroxypropyl)- $\gamma$-cyclodextrin studied by affinity capillary electrophoresis. Electrophoresis. 2020. Vol. 41(1-2), P. 112-115.

9. Sursyakova V.V., Levdansky V.A., Rubaylo A.I. Determination of binding constants for strong complexation by affinity capillary electrophoresis: the example of complexes of ester betulin derivatives with (2-hydroxypropyl)- $\gamma$-cyclodextrin. Analytical and Bioanalytical Chemistry 2020. Vol. 412(23), P. 5615-5625.

10. Sursyakova V.V., Levdansky V.A., Rubaylo A.I. Determining binding constants for $1: 1$ and 1:2 inclusion complexes of ester betulin derivatives with (2-hydroxypropyl)- $\beta$-cyclodextrin by affinity capillary electrophoresis. Electrophoresis. DOI: 10.1002/elps.202000189.

11. Levdanskii V.A., Levdanskii A.V., Kuznetsov B.N. Synthesis of betulin dibenzoate and diphthalate. Chemistry of Natural Compounds 2017. Vol. 53(2), P. 310-311.

12. Патент 2614149 РФ. Левданский В.А., Левданский А.В., Кузнецов Б.Н. Способ получения дифталата бетулинола. Опубл. 23.03.2017. [Patent 2614149 RU. Levdanskij V.A., Levdanskij A.V., Kuznetsov B.N. Method for producing betulinol diphtalate. Publ. Date 23.03.2017 (In Russ.)].

13. Патент 2638160 РФ. Левданский В.А., Левданский А.В., Кузнецов Б.Н. Способ получения дисукцината бетулинола. Опубл. 12.12.2017 [Patent 2638160 RU. Levdanskij V.A., Levdanskij A.V., Kuznetsov B.N. Method of producing betulinol disuccinate. Publ. Date 12.12.2017 (In Russ.)].

14. Le Saux T., Varenne A., Gareil P. Peak shape modeling by Haarhoff-Van der Linde function for the determination of correct migration times: A new insight into affinity capillary electrophoresis. Electrophoresis 2005. Vol. 26, P. 3094-3104.

15. Dubský P., Ördögová M., Malý M., Riesová M. CEval: All-in-one software for data processing and statistical evaluations in affinity capillary electrophoresis. Journal of Chromatography A 2016. Vol. 1445, P. 158-165. 